Journal of Computer Science 8 (1): 149-158, 2012

ISSN 1549-3636

(C) 2012 Science Publications

\title{
Optimal Network Design for Efficient Energy Utilization in Wireless Sensor Networks
}

\author{
Chutima Prommak and Sujitra Modhirun \\ School of Telecommunication Engineering, Institute of Engineering \\ Suranaree University of Technology Nakhon Ratchasima, 30000 Thailand
}

\begin{abstract}
Problem statement: This research presents a study of the optimal network design for efficient energy utilization in continuous data-gathering Wireless Sensor Networks (WSNs). We first examine the problem of minimizing the network cost through the minimum number of relay-station installation. Then we further investigate the problem of minimizing the energy consumption of the sensor nodes. Approach: We model the network design problem as an integer linear programming. Our key contribution is that the proposed models not only guarantee the network lifetime but also ensure the radio communication between the energy-limited sensor nodes so that the network can guarantee packet delivery from sensor nodes to the base station. Results: Numerical experiments were conducted to evaluate and demonstrate the effectiveness of the proposed methods in various network scenarios. Conclusion: The results demonstrate the capabilities of the proposed methods to design WSNs with more efficient energy utilization compared with other methods in term of total energy consumption and average energy consumption of SNs in the networks.
\end{abstract}

Key words: Network design, wireless sensor networks, energy efficiency, Integer Linear Programming (ILP), assignment problem, network optimization, energy consumption, energy minimization, network lifetime

\section{INTRODUCTION}

Wireless Sensor Networks (WSNs) have become potential solutions for a wide range of applications such as farm monitoring, building and factory management and military controls (Bojkovic and Bakmaz, 2008). Gathering environmental information is a common function that makes use of WSNs, in which the Sensor Nodes (SNs) are deployed in the sensing field and the Base Station (BS) is used to collect and analyze the sensing data. SNs send data to BS directly or indirectly via other intermediate $\mathrm{SN}(\mathrm{s})$. SNs usually operate by using limited energy sources such as batteries. It may be undesirable to replace or recharge SNs due to high maintenance cost. In this case, Relay Stations (RSs) are deployed (as shown in Fig. 1) to receive and forward data from SNs to BS so that the energy-limited SNs can live for a desired period of the network lifetime. RSs may equip with more sophisticated energy sources such as solar cells with larger batteries. In order to operate WSNs under efficient energy utilization of SNs, we need effective network design approaches considering practical issues such as limitation of network cost, energy and radio communication range.
Several study have devoted to the study of WSN design problems in which the energy limitation of sensor nodes is the main concern (Shi et al., 2009; Paul et al., 2010; Azad and Chockalingam, 2006; Chan et al., 2008; Luo and Hubaux, 2010; Zahariadis et al., 2009; Narayanan and Bhaskar, 2004; Levendovszky et al., 2008; Guo et al., 2008). In (Shi et al., 2009; Paul et al., 2010; Azad and Chockalingam, 2006; Chan et al., 2008), the authors present the study of the WSN design in term of the base station placement problems. Particularly, in (Shi et al., 2009; Paul et al., 2010) the objective is to maximize the network lifetime for a given number of base stations to be installed in the network. Besides optimal base station placement, in (Chockalingam, 2006; Chan et al., 2008) the authors consider determining the optimal number of base stations.

Other approaches proposed to address the network lifetime problems include optimal routing (Luo andHubaux, 2010; Zahariadis et al., 2009) and optimal rate allocation (Narayanan and Bhaskar, 2004; Levendovszky et al., 2008). Assuming the transmitting power level of sensor nodes can be adjusted based on the distance, (Luo and Hubaux, 2010) focuses on the shortest path problems to find optimal route from sensor nodes to BS.

Corresponding Author: Chutima Prommak, School of Telecommunication Engineering, Suranaree University of Technology,

Nakhon Ratchasima, 30000 Thailand Tel: 66-44-224392 


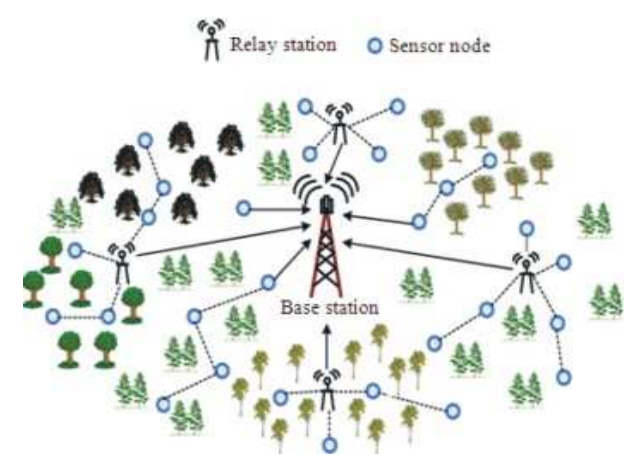

Fig. 1: Relay station deployment in WSN

Zahariadis et al. (2009) considered the remaining energy of SNs in their routing protocol. In (Narayanan and Bhaskar, 2004), the maximum data extraction problem was investigated with special consideration on the limitation of SN battery energy. Levendovszky et al. (2008) proposed the packet forwarding protocol that aims to maximize the network lifetime.

Guo et al. (2008), the authors proposed a Binary Integer Programming (BIP) for the relay node placement and assignment problems. The objective is to maximize the number of packets received at the base station and achieve a specified network lifetime. While their contribution is significant, the proposed method did not consider flow conservation constraints and could not provide packet delivery guarantee. Furthermore, the network cost was not taking into account and the number of hops between $\mathrm{SN}$ and BS is limited to two hops. For this reason, more flexible and effective approaches for the WSN design with the use of relay stations are needed.

In our study we propose a novel WSN design approach, accounting for the flow conservation and the network cost consideration in the network design process. Specifically, we aim to solve the RS placement and assignment problem for WSNs that can guarantee network lifetime and guarantee packet delivery from all SNs in the network by utilizing multiple hop RSs at the minimum network cost and minimum energy consumption.

The rest of the study is organized in four sections as followed. The next section, Materials and Methods, provides the problem definition and describes the problem formulation. Then, the Results-section report our investigation about the effects of network sizes and the distribution of SNs on the performance of the network configurations designed by the proposed model. After that the Discussion-section presents numerical comparisons and analysis of various network design scenarios. The last section, Conclusion, summarizes our research study and describes our ongoing research.

\section{MATERIALS AND METHODS}

Problem formulation:

Problem definition:The proposed WSN design in this study focuses on RS placement and assignment problems which involve selecting locations to install RSs from a set of candidate sites and determining a set of SNs and their routes to deliver sensing information to suitable RS for efficient energy utilization. Here the network configuration is formed in the way that $\mathrm{SNs}$ can communicate directly to BS or indirectly via other SNs and/or the selected RS which connects to BS. Specifically, the proposed model aims to determine the minimum number of RSs and the optimal locations to install them in the sensing field. Moreover, the proposed model aims to determine routes to deliver sensing information from a set of SNs to the suitable RS so that the resulting network configuration can guarantee the required network lifetime and ensure the radio communication between SNs so that the network can guarantee packet delivery from SNs to BS.

Here the network lifetime is defined as the duration from starting the network until the first SN depletes its battery power. This is a common definition of the WSN lifetime (Al-Turjman et al., 2009). It is assumed that the SNs are distributed across the sensing field and the sensing data can be delivered continuously through other SNs and/or RS in a multi-hop manner.

Problem formulation:The proposed WSN design problem is formulated as Integer Linear Programming (ILP) models. We propose two network design models, including the RS placement and assignment problem and the minimum energy-RS placement problem. Table 1 defines notations used in the proposed models.

RS placement and assignment problem:The RS placement and assignment problem, denoted as RPAP, aims to minimize the number of RSs and find optimal locations to install them so that the radio communication between nodes in the network and the required network operation period can be guaranteed. We incorporate the network design requirements into the following mathematical model, consisting of the objective function 1 and constraints 2-12.

\section{Objective function:}

$$
\operatorname{Minimize} \sum_{\forall j \in J} x_{j}
$$




\begin{tabular}{|c|c|}
\hline Sets & \\
\hline I & A set of Sensor Nodes (SNs) \\
\hline $\mathrm{J}$ & $\begin{array}{l}\text { A set of candidate sites to install Relay } \\
\text { Stations (RSs) }\end{array}$ \\
\hline $\begin{array}{l}\text { M } \\
\text { Decision variables }\end{array}$ & A set of Base Stations (BSs) \\
\hline $\mathrm{x}_{\mathrm{j}}$ & $\begin{array}{l}\text { A binary }\{0,1\} \text { variable that equals } 1 \text { if } \\
\text { the RSis installed at site } j ; j \in J ; 0 \text { otherwise }\end{array}$ \\
\hline$s_{\mathrm{ik}}$ & Data sent from $\mathrm{SN}$ i to $\mathrm{SN} k ; i$ and $\mathrm{k} \in \mathrm{I}$ \\
\hline & Data sent from $S N$ i to $R S j ; i \in I$ and $j \in J$ \\
\hline & Data sent from $S N$ i to $B S m ; i \in I$ and $m \in M$ \\
\hline $\mathrm{C}_{\mathrm{tSN}}$ & $\begin{array}{l}\text { Energy consumption coefficient for } \\
\text { transmitting data from sensor node } \\
\text { i to } \mathrm{SN} k ; \mathrm{i} \text { and } \mathrm{k} \in \mathrm{I}\end{array}$ \\
\hline $\mathrm{C}_{\mathrm{tRS}}$ & $\begin{array}{l}\text { Energy consumption coefficient for } \\
\text { transmitting data from sensor node } \\
i \text { to } R S j ; i \in I, j \in J\end{array}$ \\
\hline $\mathrm{C}_{\mathrm{tBS}}$ & $\begin{array}{l}\text { Energy consumption coefficient for } \\
\text { transmitting data from sensor node } \\
\text { i to } B S \mathrm{~m} ; \mathrm{i} \in \mathrm{I}, \mathrm{m} \in \mathrm{M}\end{array}$ \\
\hline $\mathrm{C}_{\mathrm{r}}$ & $\begin{array}{l}\text { Energy consumption coefficient } \\
\text { for receiving data }\end{array}$ \\
\hline $\mathrm{P}_{\mathrm{tSN}}$ & $\begin{array}{l}\text { The received signal strength } \\
\text { threshold for SNs }\end{array}$ \\
\hline $\mathrm{P}_{\mathrm{tRS}}$ & $\begin{array}{l}\text { The received signal strength } \\
\text { threshold for RSs }\end{array}$ \\
\hline $\mathrm{P}_{\mathrm{tBS}}$ & $\begin{array}{l}\text { The received signal strength } \\
\text { threshold for BS }\end{array}$ \\
\hline $\mathrm{P}_{\mathrm{ik}}$ & $\begin{array}{l}\text { The signal strength that a } \mathrm{SN} k \text { receives } \\
\text { from } \mathrm{SN} \mathrm{i} ; \mathrm{i} \text { and } \mathrm{k} \in \mathrm{I}\end{array}$ \\
\hline$P_{i j}$ & $\begin{array}{l}\text { The signal strength that a RS } j \text { receives } \\
\text { from } \mathrm{SN} i ; i \in \mathrm{I} \text { and } j \in J\end{array}$ \\
\hline $\mathrm{P}_{\mathrm{im}}$ & $\begin{array}{l}\text { The signal strength that a } B S m \text { receives } \\
\text { from } \mathrm{SN} i ; \mathrm{i} \in \mathrm{I} \text { and } \mathrm{m} \in \mathrm{M}\end{array}$ \\
\hline M & Buffer size limitation of RSs \\
\hline $\mathrm{T}$ & The required network lifetime \\
\hline$E_{i}$ & Initial energy of battery of $\mathrm{SNs}$ \\
\hline $\mathrm{g}_{\mathrm{i}}$ & Data generating rate of $\mathrm{SNs}$ \\
\hline
\end{tabular}

\section{Constraints:}

$$
\begin{aligned}
& \mathrm{s}_{\mathrm{ik}}\left(\mathrm{P}_{\mathrm{ik}}-\mathrm{P}_{\mathrm{t}_{-} \mathrm{SN}}\right) \geq 0 \quad, \forall \mathrm{i}, \mathrm{k} \in \hat{\mathrm{I}}, \mathrm{i} \neq \mathrm{k} \\
& \mathrm{r}_{\mathrm{ij}}\left(\mathrm{P}_{\mathrm{ij}}-\mathrm{P}_{\mathrm{t}_{-} \mathrm{RS}}\right) \geq 0, \forall \mathrm{i} \in \mathrm{I}, \forall \mathrm{j} \in \mathrm{J} \\
& \mathrm{b}_{\text {im }}\left(\mathrm{P}_{\text {im }}-\mathrm{P}_{\mathrm{t}_{-} \mathrm{BS}}\right) \geq 0 \quad \forall \mathrm{i} \in \mathrm{I}, \forall \mathrm{m} \in \mathrm{M} \\
& \left(T^{*} g_{i}\right)+\sum_{\forall n \in I}^{n \neq i} s_{n i}=\sum_{\forall k \in I}^{i \neq k} s_{i k}+\sum_{\forall j \in J} r_{i j}+\sum_{\forall m \in M} b_{i m}, \forall i \in I \\
& \sum_{\forall i \in I}\left(\mathrm{~T}^{*} \mathrm{~g}_{\mathrm{i}}\right)=\sum_{\forall \mathrm{j} \in \mathrm{J}} \sum_{\forall \mathrm{i} \in \mathrm{I}} \mathrm{r}_{\mathrm{ij}}+\sum_{\forall \mathrm{m} \in \mathrm{M}} \sum_{\forall i \in \mathrm{I}} \mathrm{b}_{\mathrm{im}} \forall \mathrm{j} \in \mathrm{J} \\
& \sum_{\forall i \in I} r_{i j}=M x_{j} \forall j \in J \\
& \sum_{\forall n \in I}^{n \neq i} C_{r} s_{n i}+\sum_{\forall k \in I}^{i \neq k} C_{t_{1} S N} s_{i k}+\sum_{\forall j \in J} C_{t_{-} R S} r_{i j}+\sum_{\forall m \in M} C_{t_{-} B S} b_{i m} \leq E_{1} \forall i \in I \\
& \mathrm{x}_{\mathrm{j}} \in\{0,1\} \quad, \forall \mathrm{j} \in \mathrm{J} \\
& \mathrm{s}_{\mathrm{ik}} \geq 0 \quad \forall \mathrm{i}, \mathrm{k} \in \mathrm{I}, \mathrm{i} \neq \mathrm{k}
\end{aligned}
$$

$$
\begin{aligned}
& \mathrm{r}_{\mathrm{ij}} \geq 0 \quad \forall \mathrm{i} \in \mathrm{I} \forall \mathrm{j} \in \mathrm{J} \\
& \mathrm{b}_{\mathrm{im}} \geq 0 \quad \forall \mathrm{i} \in \mathrm{I} \forall \mathrm{m} \in \mathrm{M}
\end{aligned}
$$

The objective function 1 aims to minimize the number of RSs that will be installed in the network. Constraints 2-4 ensure the radio connectivity between nodes in the network by assessing the signal strength received at each node. These constraints enforce that the received signal strength must be greater than the specified threshold. Constraint 5 is a flow balancing Eq. 1-13 of each SN in the network. It states that sensing information $\mathrm{g}_{\mathrm{i}}$ generated by $\mathrm{SN}$ i plus all incoming bits from other SNs is equal to total outgoing bits sent from $\mathrm{SN}$ i to other SNs or RSs or BS. Constraint 6 states that all sensing information generated by SNs can be sent to $\mathrm{RSs}$ or BS. It guarantees packet delivery from $\mathrm{SNs}$ to RSs or BS. Constraint 7 enforces that RS must be installed at the site $\mathrm{j}$ if a communication link between $\mathrm{SN} \mathrm{i}$ and $\mathrm{RS}$ at site $\mathrm{j}$ is established. Moreover it specifies the buffer size limitation of the RS $j$. Constraint 8specifies the energy limitation of each SN. It states that the total energy consumption (for receiving and transmitting the sensing information) at each $\mathrm{SN}$ during the required network lifetime cannot exceed the initial node energy. Constraint 9states that $\mathrm{x}_{\mathrm{j}}$ are binary $0-1$ variables. Finally, constraint $10-12$ state that $s_{i k}, r_{i j}$ and $b_{i m}$ are non-negative variables.

Minimum energy-RS placement problem: The minimum energy-RS placement problem, denoted as MERP, aims to minimize energy consumption of SNs in the network with the use of mathematical model written in 13. Furthermore, we enforce that the resulting network can guarantee the required network lifetime and the packet delivery from all $\mathrm{SNs}$ to $\mathrm{BS}$. We incorporate these network design requirements through a set of constraints 2-12 described earlier:

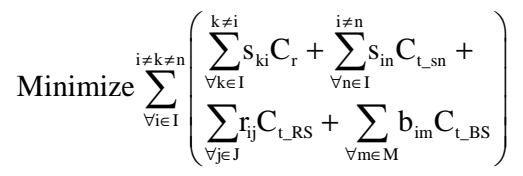

\section{RESULTS}

We present numerical study and analysis demonstrating the WSN design using the RPAP model. Particularly, we investigate the effects of network sizes and the distribution of SNs on the performance of the network configurations designed by the RPAP model.

We first describe the energy consumption model and the radio propagation model used in the experiments. Next the experiment setup and the numerical results are presented. 


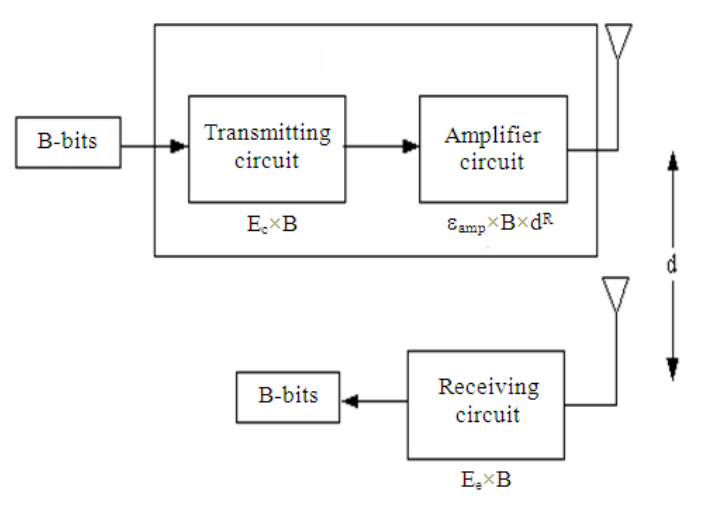

Fig. 2: Transmitting and receiving node model

Energy consumption model:In our experiments, the energy model Fig. 2 is used to compute the energy consumption for transmitting and receiving signal which are rewritten here in Eq. 14 and 15 (Ding et al., 2007; Heinzelman et al., 2000):

$\mathrm{Tx}=\left(\mathrm{E}_{\mathrm{c}} \times \mathrm{B}\right)+\left(\varepsilon_{\mathrm{amp}} \times \mathrm{B} \times \mathrm{d}^{\mathrm{n}}\right)$

$\mathrm{Rx}=\mathrm{E}_{\mathrm{c}} \times \mathrm{B}$

Where:

$\mathrm{E}_{\mathrm{c}}=$ Energy consumption in transmitting and receiving circuit (nJ/bit)

$\varepsilon_{\text {amp }}=$ Energy consumption in amplifier $\left(\mathrm{pJ} / \mathrm{bit} / \mathrm{m}^{\mathrm{n}}\right)$

$\mathrm{B}=$ Number of bits (bits)

$\mathrm{d}=$ Distance between transmitter and receiver $(\mathrm{m})$

$\mathrm{n} \quad=$ Index path-loss exponent

Radio propagation model:It is necessary to compute the received signal strength at SNs, RSs and BS and input the obtained values in the RPAP model to find the optimal locations to install RSs. This computation can be done by using the propagation model. In this study we use the Simplified path-loss model to evaluate the path loss in WSN (Goldsmith, 2005).

The received signal strength at SNs, RSs and BS $\left(\mathrm{P}_{\mathrm{ik}}, \mathrm{P}_{\mathrm{ij}}\right.$ and $\left.\mathrm{P}_{\mathrm{im}}\right)$ are pre-computed by using the simplify path loss model presented in (Goldsmith, 2005) and it is written here in Eq. 16. The pre-computed values are input into the RPAP model to find the optimal locations to install RSs:

$$
\begin{aligned}
& P_{r}=P_{t}+K-10 n \log _{10}\left[\frac{d}{d_{0}}\right] \\
& K(d B)=20 \log _{10} \frac{\lambda}{4 \pi d_{0}}
\end{aligned}
$$

Where:

$P_{r}=$ The received signal strength $(\mathrm{dBm})$

$\mathrm{P}_{\mathrm{t}}=$ The transmit power $(\mathrm{dBm})$

$\mathrm{n}=$ The index path loss exponent

$\mathrm{d}=\mathrm{A}$ distance between the transmitting node and the Receiving node $(\mathrm{m})$

$\mathrm{d}_{0}=$ A reference distance for the antenna far field $(\mathrm{m})$

$\lambda=$ The signal wavelength (m)

Experiment setup: In numerical experiments, we consider the sensing field of size $500 \times 500 \mathrm{~m}$ in which one base station is located in the middle. To observe the effects of the network sizes (the number of $\mathrm{SNs}$ ) on the energy consumption of the networks, we consider three different network sizes which represent the real network in practice, including the networks of size 30, 50 and 80 SNs. Figure 3-5 show the considered network scenarios, in which 110 candidate sites to install RSs are denoted with the symbol ' + '. The network lifetime of $800 \mathrm{sec}$ is considered here for the preliminary network design experiments.

We consider the WSN standards IEEE 802.15.4 in the numerical experiments. Table 2 shows the parameters used in the numerical experiments (Levendovszky et al., 2008; Guo et al., 2008) for more details. The received signal strength threshold to ensure the radio connectivity between nodes in the network is set to- $90 \mathrm{dBm}$.

In the experiment, we input the set of SNs, their locations, the required network lifetime and other parameters to the RPAP model. We then solve the WSN design by implementing the RPAP model with the ILOG-OPL development studio and solving with CPLEX 5.2 optimization solver. Computations are performed on an Intel Centrino Core2 Duo Processor $2.0 \mathrm{GHz}$ and $2 \mathrm{~GB}$ of RAM.

Numerical results and analysis: Figure 6 shows the cumulative distribution function comparing the average energy consumption in the networks of different sizes. It can be observed that as the network sizes enlarge (in term of the number of SNs used in the sensing field), the energy consumption of the sensor node increases.In the case of the network size of $30 \mathrm{SNs}, 80 \%$ of SNs consume energy less than 32 joules whereas the energy consumption of $80 \%$ of SNs in the network size of 50 and $80 \mathrm{SNs}$ are 39 and 66 joules, respectively. 


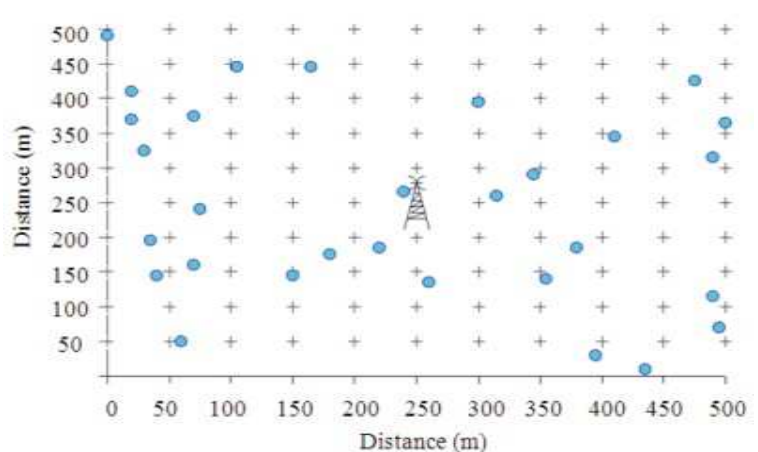

(a)

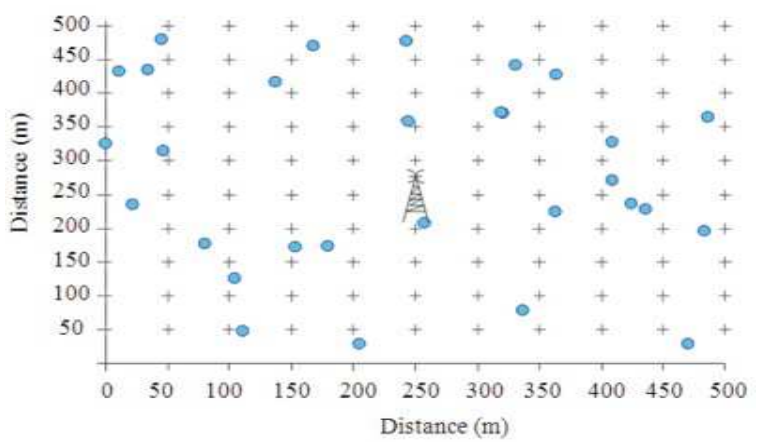

(b)

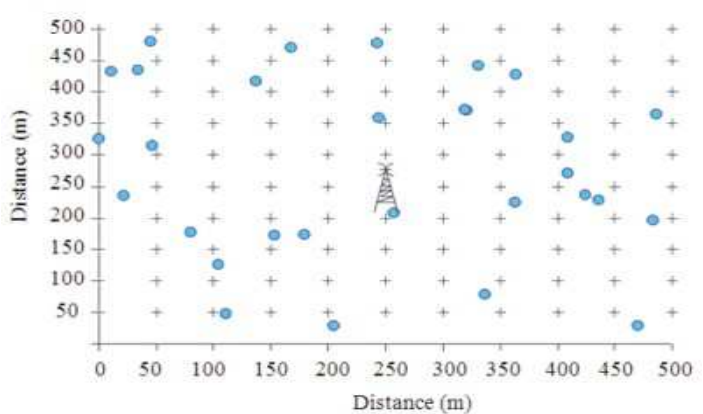

(c)

Fig. 3: Network scenarios of 30 sensor nodes (a) Network 30A (b) Network 30B (c) Network 30C

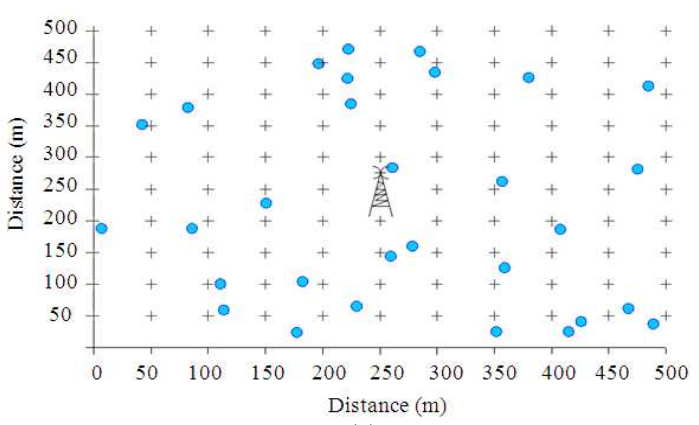

(a)

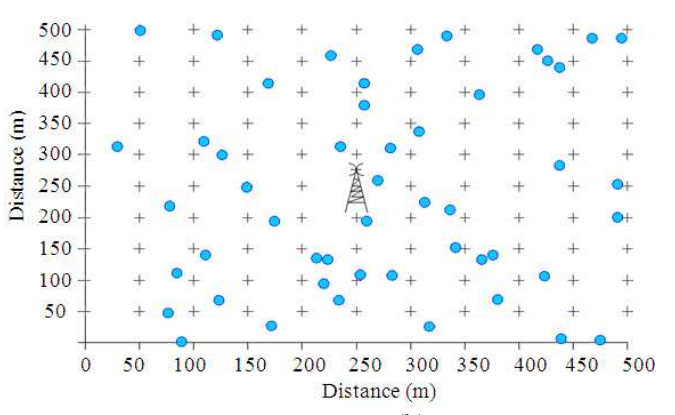

(b)

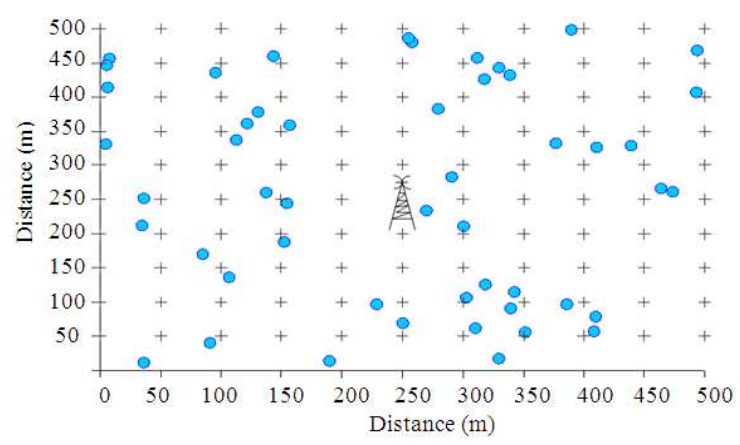

(c)

Fig. 4: Network scenarios of 50 sensor nodes (a) Network 50A (b) Network 50B (c) Network 50C 


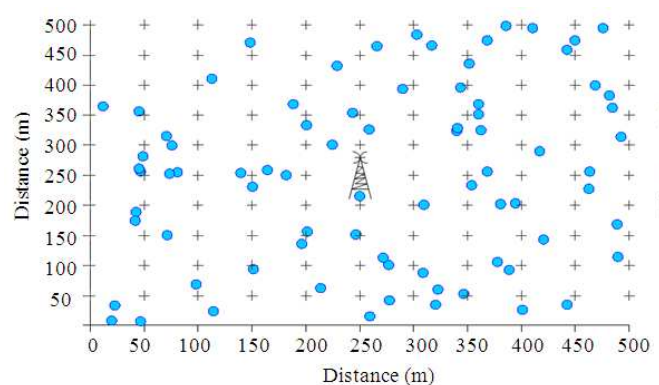

(a)

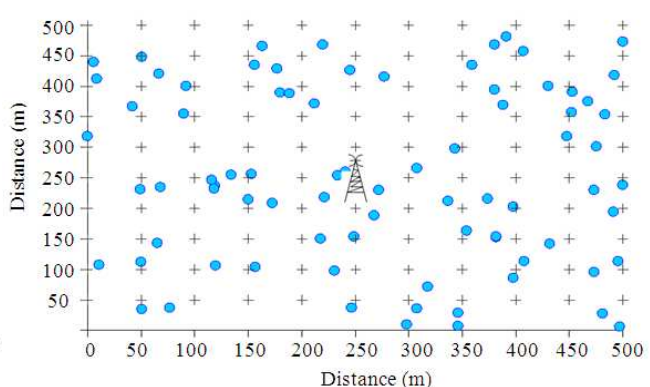

(b)

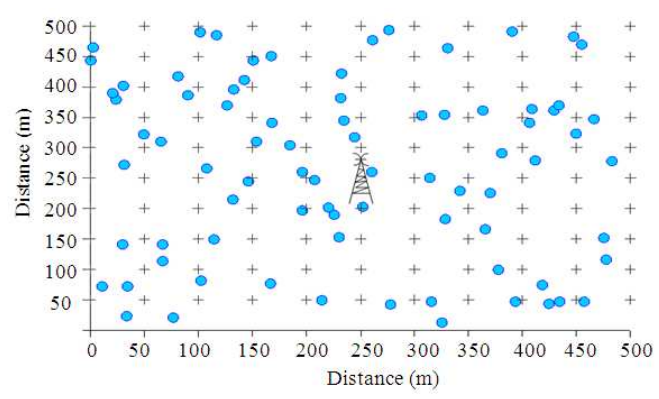

(c)

Fig. 5: Network scenarios of 80 sensor nodes (a) Network 80A (b) Network 80B (c) Network 80C

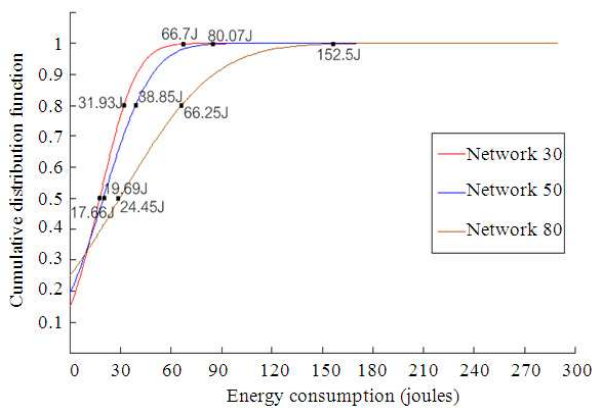

Fig. 6: Comparison of energy consumption in difference network sizes (when using RPAP model)

Table 2: Parameters used in numerical experiments

\begin{tabular}{ll}
\hline Parameters & Value \\
\hline Receiver sensitivity threshold & $-90 \mathrm{dBm}$ \\
Communication data rate & $2.5 \mathrm{kbps}$ \\
Operating frequency & $2.4 \mathrm{GHz}$ \\
Initial energy of sensor nodes & 35,160 joules \\
Buffer size at relay stations & $10,000,000$ bits \\
Transmit power & $32 \mathrm{~mW}$ \\
Reference distance & $1 \mathrm{~m}$. \\
Path loss exponent & 4 \\
\hline
\end{tabular}

Figure 7-9 illustrate the cumulative distribution functions of energy consumption in different networks sizes consisting of 30, 50 and $80 \mathrm{SNs}$, respectively. Each figure shows the CDF graph of the energy consumption of SNs in the networks of the same size (denoted as network $\mathrm{A}, \mathrm{B}$ and $\mathrm{C}$, in which locations of SNs are varied).

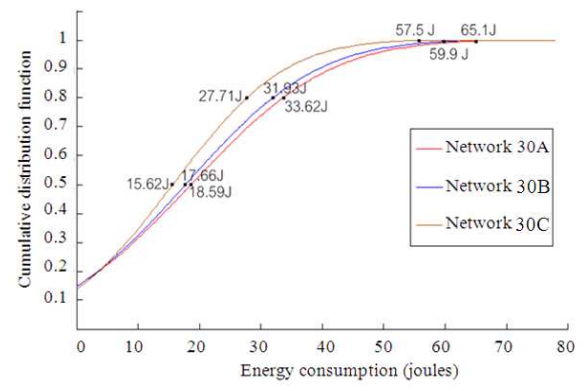

Fig. 7: Cumulative distribution function of the energy consumption in networks of 30 SNs (using RPAP)

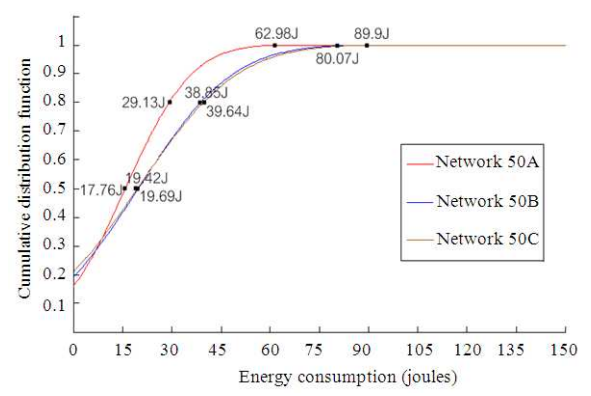

Fig. 8: Cumulative distribution function of the energy consumption in networks of $50 \mathrm{SNs}$ (using RPAP)

We can see that for each network size, as locations of SNs change, the energy consumption of SNs in the network remains almost the same. 


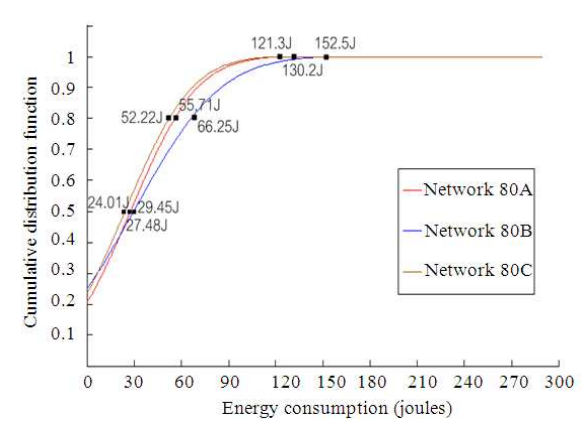

Fig. 9: Cumulative distribution function of the energy consumption in networks of 80 SNs (using RPAP)

The reason is that each $\mathrm{SN}$ has to send its sensing data to BS either directly or indirectly via neighbour SNs or RS. Thus the average energy consumption of SNs depends mainly on the number of SNs in the sensing field.

\section{DISCUSSION}

We present discussion and analysis demonstrating the WSN design using the proposed RPAP and MERP model. We compare our model with those presented in (Shi et al., 2009) of which the objective function is to maximize the network lifetime with constraints on SNs' battery energy and RSs are not used. We call it a MNL (Maximize Network Lifetime) model. RPAP model, on the other hands, aims to minimize the RS installation cost while maintaining the required period of the network lifetime. We incorporate the path loss function in the constraints where we calculate the received signal strength to guarantee the sufficient signal strength that can ensure the radio communication between nodes in WSNs. Moreover, we enforce the flow conservation rule to guarantee packet delivery from SNs to BS.

Here we consider two version of MERP. MERP1 aims to minimize the energy consumption of SNs in the network and guarantee the required period of the network lifetime as described in the problem formulation.

Another version, MERP2, also aims to minimize the energy consumption and guarantee the required period of the network lifetime. In MERP2, We apply an additional constraint on the number of RSs that can be used in the network to take into account the network budget limitation. The following describe the experimental setup and present numerical comparisons and analysis.

Experiment setup:In numerical experiments, we consider the sensing field of size $500 \times 500 \mathrm{~m}$ and consider three different network sizes consisting of 30 , 50 and 80 SNs as shown in Fig. 3-5, respectively. There are 110 candidate locations to install RSs which are represented by the symbol ' + '.
Here the WSN standards IEEE 802.15.4 are considered in the numerical experiments as well. Table 2 shows the parameters used in the numerical experiments ((Levendovszky et al., 2008; Guo et al., 2008) for more details).

First of all, MNL model is used to derive the maximum network lifetime with constraints on $\mathrm{SNs}$ ' battery energy for the case that no RS is deployed. We apply the SN's initial energy of 61,560 joules which is computed by considering the use of four AA batteries and the current consumption of 0.1 amps for the duration of $10^{5} \mathrm{sec}$. We obtained the network lifetime of $13,825 \mathrm{sec}$ and set this value as the required network lifetimes for the WSN design by using other models.

We input the set of RS candidate sites, the required network lifetime and other parameters to the RPAP, MERP1 and MERP2 model and solve the WSN design by implementing the proposed models with the ILOG-OPL development studio and solving with CPLEX 5.2 optimization solver. Computations are performed on an Intel Centrino Core2 Duo Processor $2.0 \mathrm{GHz}$ and $2 \mathrm{~GB}$ of RAM.

Numerical comparisons and analysis:Table 3 shows numerical results comparing the number of relay stations used in the network and total energy consumption of the network designed by MNL, RPAP, MERP1 and MERP2 model for the network size of 30, 50 and 80 SNs. We can see that MNL results in highest energy consumption whereas in case of RPAP, MERP1 and MERP2, SNs use much less energy. The reason is that MNL does not deploy RS and the intermediate SNs use lot of energy to receive and forward sensing data to BS. We can observe that the minimum energy consumption is in the case of using MERP1 in which the number of installed RSs is highest. In case of MERP2, the energy consumption is higher than that of MERP1. Comparing the energy consumption of RPAP and MERP2, we can see that MERP2 could improve the energy efficiency in the network that uses the same number of RSs as that used in RPAP model. The reason is that the objective of MERP2 is to minimize network energy consumption whereas the objective of RPAP aims to minimize network cost (i.e., the number of RSs).

Table 4 shows maximum and minimum energy consumption of SNs in the networks of different sizes. We can see that MNL results in highest energy consumption for both maximum and minimum values compared with those of other methods whereas MERP1 results in the lowest energy consumption for both the maximum and minimum values. Comparing the values of RPAP and MERP2, we can see that the maximum energy consumption of MERP2 is less than that of the RPAP. 
Table 3: Number of relay stations installed in the sensing field and total energy consumption

\begin{tabular}{llc}
\hline $\begin{array}{l}\text { Network } \\
\text { design models }\end{array}$ & $\begin{array}{l}\text { Number of relay } \\
\text { stations installed }\end{array}$ & $\begin{array}{l}\text { Total energy } \\
\text { consumption of all } \\
\text { sensor nodes (joules) }\end{array}$ \\
\hline Network 30 & & \\
MNL & - & $1,514,307$ \\
RPAP & 16 & 12,621 \\
MERP1 & 27 & 3,628 \\
MERP2 & 16 & 7,254 \\
Network 50 & & \\
MNL & - & $2,748,142$ \\
RPAP & 17 & 27,014 \\
MERP1 & 37 & 5,865 \\
MERP2 & 17 & 15,405 \\
Network 80 & & \\
MNL & - & $3,295,265$ \\
RPAP & 17 & 75,661 \\
MERP1 & 59 & 9,099 \\
MERP2 & 20 & 26,575 \\
\hline
\end{tabular}

Table 4: Maximum and minimum energy consumption of a sensor node in the networks

\begin{tabular}{lrc}
\hline $\begin{array}{l}\text { Network design } \\
\text { models }\end{array}$ & $\begin{array}{l}\text { Maximum energy } \\
\text { consumption (joules) }\end{array}$ & $\begin{array}{l}\text { Minimum energy } \\
\text { consumption (joules) }\end{array}$ \\
\hline Network 30 & & \\
MNL & 61,560 & 329 \\
RPAP & 1,185 & 82 \\
MERP1 & 180 & 84 \\
MERP2 & 697 & 84 \\
Network 50 & & \\
MNL & 61,560 & 68 \\
RPAP & 2611 & 83 \\
MERP1 & 379 & 83 \\
MERP2 & 1,185 & 83 \\
Network 80 & & \\
MNL & 61,560 & 186 \\
RPAP & 8,341 & 82 \\
MERP 1 & 413 & 82 \\
MERP 2 & 1400 & 83 \\
\hline
\end{tabular}

Table 5: Average energy consumption of sensor nodes in the networks and standard deviation

\begin{tabular}{lcr}
\hline $\begin{array}{l}\text { Network design } \\
\text { models }\end{array}$ & $\begin{array}{l}\text { Average energy } \\
\text { consumption (joules) }\end{array}$ & SD (joules) \\
\hline Network 30 & & \\
MNL & 50,476 & 20,902 \\
RPAP & 420 & 259 \\
MERP1 & 120 & 26 \\
MERP2 & 241 & 159 \\
Network 50 & 54,962 & 16,722 \\
MNL & 540 & 539 \\
RPAP & 117 & 60 \\
MERP1 & 308 & 267 \\
MERP2 & & \\
Network 80 & 41,190 & 212,105 \\
MNL & 945 & 1,359 \\
RPAP & 113 & 50 \\
MERP1 & 332 & 288 \\
MERP2 & &
\end{tabular}

Table 5 shows average energy consumption of sensor nodes and standard derivation of energy consumption. We can see that MNL results in highest average energy consumption and highest standard deviation whereas MERP1 results in lowest average energy consumption and lowest standard deviation.

Figure 10-12 show effects of different network design models on the energy consumption in network of size 30, 50 and $80 \mathrm{SNs}$, respectively. The cumulative distribution function in Fig. 10 shows that RPAP results in highest energy consumption of SNs. We can see that in the network consisting of $30 \mathrm{SNs}, 80 \%$ of $\mathrm{SNs}$ consume up to 639.4 joules per node whereas those of MERP1 and MERP2 model consume up to 143.9 and 375.8 joules per node, respectively. The reason is that RPAP used the objective function that minimized the number of RSs to achieve the required network lifetime for a given amount of initial energy of SNs. On the other hand MERP1 aims to minimize energy consumption and find sufficient number of RSs to achieve the required network lifetime. So, MERP1 results in lowest energy consumption. As for MERP2, it aims at minimize energy consumption by using the same number of RSs as used in RPAP model. We can see that although using the same number of RSs, MERP2 consume less energy than that of RPAP. The reason is that MERP2 uses the objective function that minimized the energy consumption for a given number of RSs to achieve the required network lifetime. Fig. 11 and 12 show similar trend of energy consumption of different models in network size of 50 and 80 SNs.

Figure 13-15 show effects of network sizes on the energy consumption when using MERP1, MERP2 and RPAP model, respectively. The cumulative distribution functions show that in case of MERP1, network sizes have little effects on the energy consumption whereas in case of RPAP, network sizes affect the energy consumption of each node in higher degree. From Fig. 13, we can see that energy consumption of SNs in network sizes of 30, 50 and 80 SNs are not that much different. The reason is that MERP1 aims to minimize energy consumption and find sufficient number of RSs to be used in the network. In this case there is no limit on the number of RSs. Thus, the energy consumption of SNs could be compromised by the utilization of RSs.

Figure 14 and 15 show higher degree of the effects of network sizes. These two figures show that the bigger the network size (i.e., the network consisting of more number of SNs) the higher level of energy consumption of each node in the network. The reason is that RPAP and MERP2 put restriction on the number of installed RSs. Thus, as the number of SNs in the network increases, intermediate $\mathrm{SNs}$ have to receive and forward more sensing data to RSs and/or BS. 


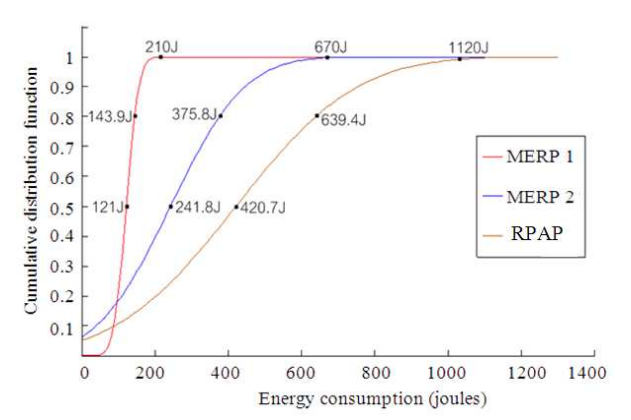

Fig. 10: Effects of different network design models on the energy consumption in network of $30 \mathrm{SNs}$

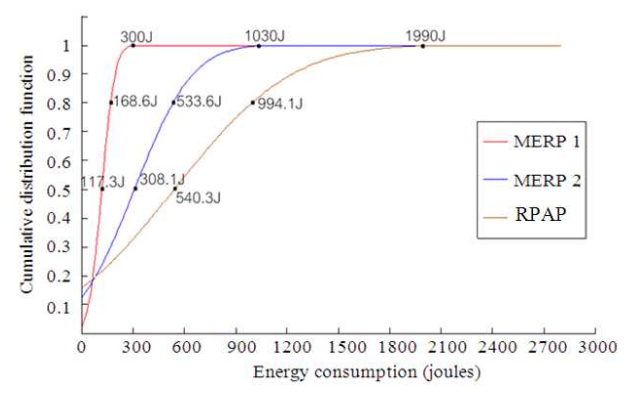

Fig. 11: Effects of different network design models on the energy consumption in network of $50 \mathrm{SNs}$

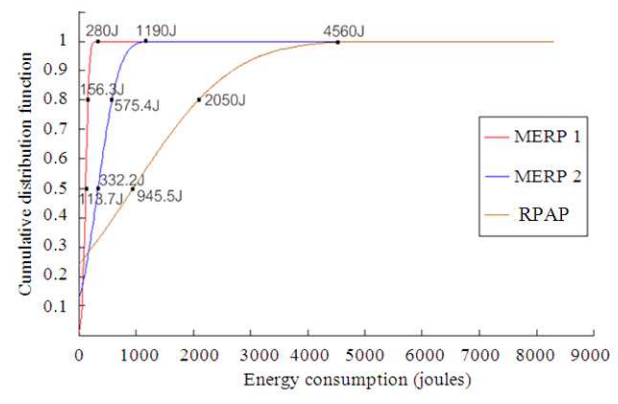

Fig. 12: Effects of different network design models on the energy consumption in network of $80 \mathrm{SNs}$

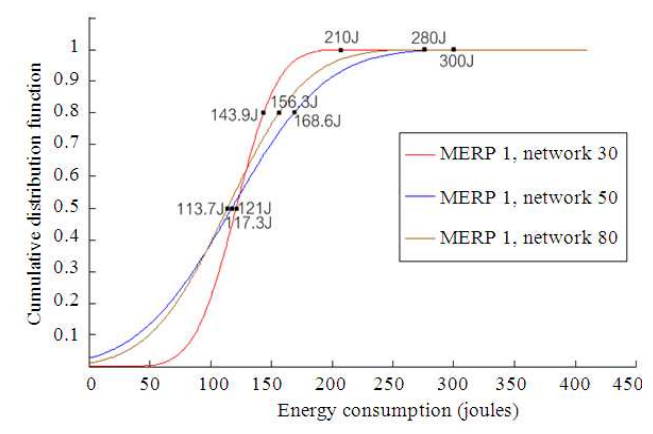

Fig. 13: Effects of network sizes on the energy consumption when using MERP1

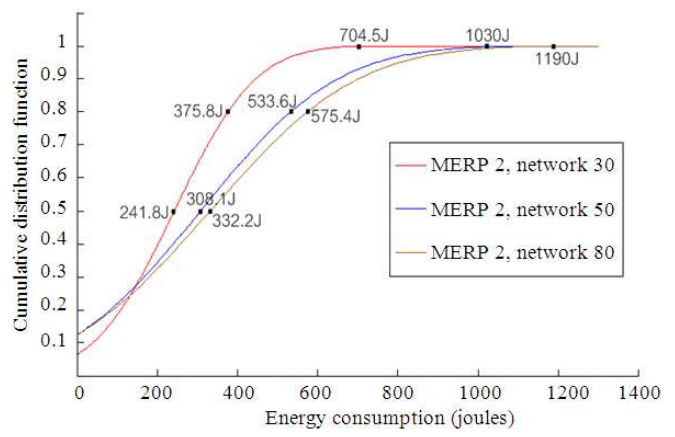

Fig.14: Effects of network sizes on the energy consumption when using MERP2

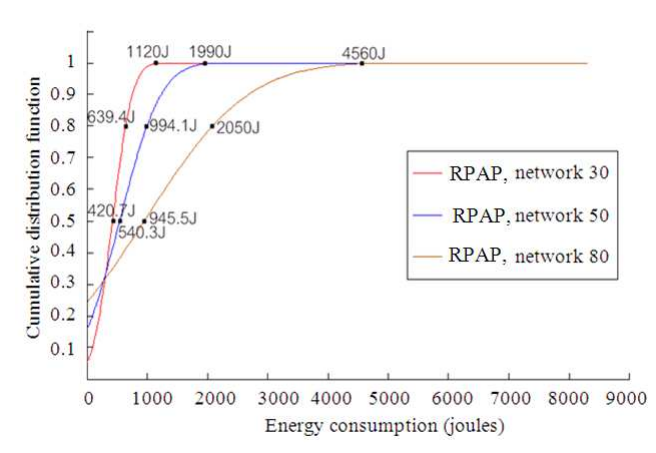

Fig. 15: Effects of network sizes on the energy consumption when using RPAP

\section{CONCLUSION}

In this study, the optimal network design for efficient energy utilization in continuous data-gathering Wireless Sensor Networks (WSNs) is investigated. Given location of Base Station (BS) and Sensor Nodes (SNs) with specified sensing rate, we propose integer linear programming models for two important problems of the WSN design, including the relay station placement and assignment problem and the minimum energy-RS placement problem.

The proposed network design models determine the optimal number and locations of Relay Stations (RSs) and the flow assignment from SNs to BS with constraints on radio communication range of SNs and the required network lifetime. Various numerical experiments were conducted to investigate the effects of network sizes and the distribution of SNs on the performance of the designed network configurations. In addition, we studied the effects of different network design models on the energy consumption of SNs in the network.Numerical experiments show that the proposed models yield WSNs with more efficient energy utilization compared with other methods in term of total energy consumption and average energy consumption of SNs in the network. 
Our ongoing study investigate the diversity of the data transmission paths to enhance the quality of delivery in noisy environments, considering the real sensor networks deployment scenarios.

\section{ACKNOWLEDGMENT}

This study was supported in part by Suranaree University of Technology (SUT), the Office of the Higher Education Commission under NRU project of Thailand and the National Research Council of Thailand (NRCT).

\section{REFERENCES}

Bojkovic, Z. and B. Bakmaz, 2008. A survey on wireless sensor networks deployment. J. Wseas Trans. Commun., 7: 1172-1181.

Shi,Y.,Y.T. Hou and A. Efrat, 2009. Algorithm design for a class of base station location problems in sensor networks. Wireless Network, 15: 21-38. DOI: 10.1007/s11276-007-0020-2

Paul, B., M.A. Matin, M.J. Showkat and Z. Rahman, 2010. Optimal placement of base stations in a two tiered wireless sensor network. J. Wseas Trans. Commun., 9: 43-52.

Azad, A.P. and A. Chockalingam, 2006. Mobile base stations placement and energy aware routing in wireless sensor networks. Proceeding of IEEE Wireless Communications and Networking Conference, Apr. 3-6, IEEE Xploor Press, Las Vegas, pp: 264-269. DOI: 10.1109/WCNC.2006.1683475

Chan, T.J., C.M. Chen,Y.F. Huang, J.Y. Lin and T.R. Chen, 2008.Optimal cluster number selection in ad-hoc wireless sensor networks. J. Wseas Trans. Commun., 7: 837-846.

Luo, J. and J.P. Hubaux, 2010. Joint sink mobility and routing to maximize the lifetime of wireless sensor networks: The case of constrained mobility. IEEE/ACM Trans. Network, 18: 871-884. DOI: 10.1109/TNET.2009.2033472

Zahariadis, T., H.C. Leligou, S. Voliotis, S. Maniatis and P. Trakadas et al., 2009. Energy-aware secure routing for large wireless sensor networks. J. WSEAS Trans. Commun., 8: 981-991.
Narayanan, S. and K. Bhaskar, 2004. Maximizing data extraction in energy-limited sensor networks. Procedings of the 23rd Annual Joint Conference of the IEEE Computer and Communications Societies INFOCOM, Mar. 7-11, IEEE Xploor Press, Hong Kong, pp: 1717-1727. DOI: 10.1109/INFCOM.2004.1354583

Levendovszky, J., A. Bojarszky, B. Karlocai and A. Olah, 2008. Energy balancing by combinatorial optimization for wireless sensor. J. WSEAS Trans. Commun., 7: 27-32.

Guo,W., X. Huang,W. Louand and C. Liang, 2008. On relay node placement and assignment for two-tiered wireless networks. Mobile Network Applied, 13: 186-197. DOI: 10.1007/s11036-008-0027-7

Al-Turjman, F.M., H.S. Hassanein and M.A. Ibnkahla, 2009. Connectivity optimization with realistic lifetime constraints for node placement in environmental monitoring. Proceedings of the IEEE 34th Conference on Local Computer Networks, Oct. 20-23, IEEE Xploor Press, Zurich, pp: 617-624. DOI: 10.1109/LCN.2009.5355140

Ding, E.J., C.N. Wang and Q. Zhou, 2007. Wireless model and deployment of sensor networks in the mine. Proceeding of the International Conference on Information Acquisition, July 8-11, IEEE Xploor Press, Seogwipo, pp: 538-542. DOI: 10.1109/ICIA.2007.4295791

Heinzelman, W.R., A. Chandrakasan and H. Balakrishman, 2000. Energy-efficient communication protocol for wireless microsensor networks. Proceeding of the 33rd Hawaii International Conference on System Sciences, Jan. 4-7, IEEE Xploor Press, USA., pp: 10-10. DOI: 10.1109/HICSS.2000.926982

Goldsmith, A., 2005. Wireless Communications. 1st Edn., Cambridge University Press, Cambridge, U.K., ISBN: 0521837162, pp: 644. 